\title{
Ethique et déontologie dans le processus de professionnalisation des coachs
}

\author{
Geneviève Guilhaume, Maître de conférences, \\ Université Bordeaux-Montaigne - Laboratoire MICA, \\ genevieve.guilhaume@u-bordeaux-montaigne.fr
}




\section{Résumé}

Dans cet article, nous analysons le rôle de l'éthique et de la déontologie dans le coaching, sa contribution à la professionnalisation des coachs et des managers. Le coaching est considéré comme un dispositif communicationnel destiné à transformer les managers en communicants pour de meilleures performances économiques. Les codes de déontologie développés par les associations de coachs sont destinés à promouvoir et à légitimer la profession. A partir des concepts de Ricœur, on peut montrer l'existence d'une éthique de soi dans le coaching, qui, par ses valeurs morales, légitimerait l'action sur la psyché dans le cadre d'une relation de confiance, qui vise à imputer à l'individu la responsabilité de ses actes ; le coach se doit d'intégrer un idéal du Moi, auquel s'identifie le manager coaché. Cette éthique de soi ne se traduit pas toujours par un pouvoir d'agir, les initiatives des managers se retrouvant prises dans des injonctions paradoxales. Le coaching transmettrait bien aux managers un pouvoir de communication, qui consisterait à euphémiser le pouvoir de décision économique du sommet, rendant parfois les coachs et les salariés « responsables » de choix imposés. L'éthique du coaching dépend donc de la sagesse pratique des coachs, issue des convictions et de l'expérience personnelle.

Mots-clé : éthique, déontologie, éthique de soi, idéal du Moi, euphémismes, sagesse pratique, professionnalisation

\footnotetext{
Abstract

In this article, we are offering an analysis of the role of ethics and deontology inside coaching and its contribution to coaches and managers guidance. Coaching is seen as a communicational program, intended to transform managers into communicators, for best economical performances. Deontological codes of coaches' associations are meant to promote their profession and have it recognized. Based on Ricoeur's concepts, we can show the existence of a moral code of ethic in the coaching process, which through its moral values, would justify a work on the psyche within the framework of a trust relationship, which aims at giving responsibilities to the individual; the coach must integrate an «ideal of myself », to which the coached manager identifies. This self ethic isn't always expressed by an empowerment, as the managers' initiatives are being caught in paradoxical orders. Coaching then would pass on to the managers a power of communication, which would consist in euphemizing the economical power of top, so that the coaches and the employees feel being « responsible » of the choices imposed upon them. Ethical coaching depends, on coaches" "practical wisdom », issued from convictions and personal experience.

Key words : ethic, deontology, self ethic, ideal of myself, euphemisms, practical wisdom professionalization
} 
Nous avons montré dans nos écrits antérieurs (Guilhaume, 2009) que le coach est un communicant d'entreprise : les objectifs du coaching résident dans un accompagnement visant à transformer la communication des managers (plus précisément leurs comportements communicationnels), à transformer leur management d'équipe ou de groupes de projet, dans le cadre d'entreprises flexibles où le travail de plus en plus complexe nécessite une coopération, donc une communication d'intercompréhension (Zarifian, 2000). Les objectifs du coaching, comme l'indique celui qui est considéré comme le «père » du coaching en France sont donc bien d'apprendre à «mieux communiquer et méta-communiquer » (Lenhardt, 2010). Le dispositif de coaching est lui-même communicationnel, puisqu'il est fondé sur la relation de feed-back qui consiste, par une maïeutique, par un retour sur soi de l'individu sollicité par le coach, à faire émerger des solutions en termes d'actions et résoudre ainsi des problèmes, qui touchent tant aux savoir-faire du manager qu'à ses attitudes et comportements. Enfin, les méthodes, empruntant aux fondements scientifiques psychologiques des thérapies brèves comportementales (programmation neuro-linguistique, analyse transactionnelle, gestalt) peuvent être perçues comme des « technologies de la communication et du changement » (Brunel, 2008)

L'analyse du rôle de l'éthique et de la déontologie dans la professionnalisation des coachs, l'analyse des résultats de cette mise en œuvre d'une éthique et d'une déontologie dans le processus de coaching notamment sur les décisions prises par les managers peuvent éclairer à la fois sous un angle plus restreint, mais aussi dans un cadre plus large, les questions autour de l'éthique des communicants.

En effet, le coach peut être considéré comme un communicant d'entreprise qui intervient en complément ou en substitut des professionnels de la communication interne. Pour V. Brûlois et J.M. Charpentier (2013), « les années 2000 peuvent être considérées comme des années difficiles pour la communication »; en effet on observe une insatisfaction croissante des salariés renvoyant aux non-dits des stratégies énoncées (p. 31). La communication tendue vers la question de l'image ou de la réputation de l'entreprise (à travers les médias numériques notamment) délaisse le lien social dans l'entreprise, le « changement vu comme une relation, un mouvement en commun » (p. 84). Or, le savoir communiquer est devenu une composante essentielle dans un univers de services, où les aléas, les évènements, les pannes et autres dysfonctionnements demandent une capacité à échanger pour résoudre des problèmes (p. 35). Ainsi, « l'écart est grand entre la communication d'interaction, vécue par les salariés dans le travail, et, le régime surplombant de la communication d'entreprise, tendue vers l'image ». Pour les auteurs, les managers eux-mêmes « à la peine », face aux contraintes gestionnaires, et « à distance» (p. 84), car ils se replieraient sur le métier, négligeraient la communication directe dans le travail : c'est là qu'intervient alors le coach pour rendre le manager plus communicant. Au niveau de l'entreprise, le coaching signifie bien l'ère d'un néo-management fondé sur l'individualisation des 
rapports de travail, sur la responsabilisation de l'individu (l'empowerment) dans tous les domaines de sa vie. Il sacralise la toute puissance de la communication dans la figure du coach, du manager, du salarié communicant (Guilhaume, 2009). Le coach intervient donc pour renforcer la professionnalisation des communicants, les communicants incluant, dans cette perspective, à côté des « responsables de com », managers et salariés. L'éthique et la déontologie dans le coaching participent donc bien directement et indirectement aux questionnements sur la professionnalisation des communicants d'entreprise.

Précisons notre cadre théorique et méthodologique : à partir d'une vaste enquête de thèse ${ }^{1}$ conduite sous la forme d'entretiens semi-directifs, nous avons analysé le coaching comme un dispositif communicationnel paradoxal, contribuant à l'intériorisation par les coachés des paradoxes du management et participant à une violence symbolique s'imposant aux managers et aux salariés. Nous avons également montré, notamment à partir des approches critiques de P. Bourdieu sur le langage et le pouvoir symbolique (Bourdieu, 2001), que la communication du coaching était euphémisée, mettant en avant une théorie du «gagnant-gagnant », masquant bien souvent des rapports de force défavorables aux managers et aux salariés (Guilhaume, 2009).

Dans ce cadre (et sans préjuger de l'évolution de notre cadre théorique), nous souhaiterions maintenant résoudre l'énigme suivante : pourquoi, dans la restitution de leurs pratiques, les coachs font-ils systématiquement état de leur souci éthique, en évoquant très peu la déontologie ? Ceci nous conduit alors à explorer trois hypothèses :

- Les codes de déontologie seraient un outil de promotion du coaching

- L'éthique de soi dans le coaching fonderait la performance du dispositif, à l'ère du néo-management. Elle s'appuierait sur une approche très personnelle des coachs, à l'origine du flou de la professionnalisation

- Les paradoxes de cette éthique de soi conduiraient à doter le manager du pouvoir d'agir dans les discours, qui serait plutôt, dans les pratiques, un pouvoir de communiquer déresponsabilisant les organisations, manifestant les limites de la professionnalisation des coachs.

Notre recherche s'appuie, d'une part, sur l'étude des codes et chartes déontologiques des associations professionnelles de coachs, notamment SFCoach - Société Française de Coaching - et ICF - International Coaching Federation France - (les discours du

\footnotetext{
${ }^{1}$ Les dispositifs de coaching et de formation expérientielle destinés aux managers-communicateurs, enjeux communicationnels et violence symbolique dans l'entreprise, Université Paris 13, 2006
} 
coaching) et sur l'actualisation de notre enquête (les pratiques du coaching) réalisée par deux projets tutorés d'étudiants de master 1 que j'ai dirigés : le premier2 en 2011 sur le coaching externe, le deuxième 3 en 2012 sur les coachings externes-internes. Ces entretiens 4 comportaient des questions et relances sur la professionnalisation des coachs, l'éthique et la déontologie. C'est l'analyse de l'écart entre discours (officiels) du coaching et pratiques des coachs qui nous a progressivement amenée à nos principaux résultats de recherche.

\section{Ethique et déontologie}

L'éthique renvoie à un concept philosophique. P. Ricoeur réserve le terme d'éthique à «la visée de la vie bonne, avec et pour les autres, dans des institutions justes » (Ricoeur, 1990). Pour l'auteur, il est nécessaire de soumettre la visée éthique à l'épreuve de la norme : il s'agit alors d'un point de vue déontologique (déontologie signifiant « devoir »). Plus précisément, le philosophe désigne une éthique antérieure à la morale de l'obligation, ou éthique fondamentale, et des éthiques postérieures visant à insérer les normes dans des situations concrètes : les codes déontologiques seraient donc ces dispositifs pratiques, caractéristiques des éthiques postérieures ou appliquées, selon Ricoeur.

La déontologie du coaching est donc un ensemble de règles et de devoirs qui régissent la profession, la conduite de ceux qui l'exercent et les rapports entre ceuxci et leurs clients et leur public. Assortis de possibles sanctions, les codes de déontologie promeuvent la profession, à côté des offres de certification des coachs de niveaux différents, de la supervision et bien sûr des référentiels de compétences. Ainsi, la société française de coaching indique bien que

Le code est opposable à tout membre de la Société Française de Coaching. Il vise à formuler des points de repère déontologiques, compte tenu des spécificités du coaching en tant que processus d'accompagnement d'une personne dans sa vie professionnelle. Ce code de déontologie est donc l'expression d'une réflexion éthique ; il offre des principes généraux dont l'application pratique requiert une capacité de discernement (www.sfcoach.org, consulté le 27 avril 2016).

Le professionnalisme des coachs est un sujet abordé dans tous les entretiens :

Le professionnalisme porte sur son comportement personnel, deuxièmement, sur une bonne connaissance des profils de manager et de cadre, bonne connaissance des profils professionnels troisièmement, bonne connaissance des organisations et

\footnotetext{
${ }^{2}$ huit consultants-coachs externes

${ }^{3}$ quatre coachs externes, deux coachs internes et quatre managers coachés

${ }^{4}$ Méthode de l'entretien compréhensif en sociologie de l'action publique (Pinson \& Sala Pala, 2007)
} 
des entreprises. C'est ça, son professionnalisme... et d'une manière très opérationnelle (entretien coach externe 2011).

Ceci correspond aux référentiels de compétences des associations de coaching. Ainsi, le référentiel de la SFCoach élaboré avec un universitaire (M. Vial, Université d'Aix-en-Provence) en 2005 propose sept domaines de compétences : elles sont déclinées sous forme de verbes d'action, comme «travaille la demande », « utilise les modalités de la communication», «accompagne le coaché dans son changement », comme le montre le schéma figurant en annexe. La réalisation de ces actions exige des «savoirs et références", ainsi qu'un «travail sur soi et une expérience ». On observe dans ce référentiel l'importance de l'appui scientifique, mais aussi la déclinaison de compétences très proches de celles des cartographies de métiers des entreprises (savoirs, savoir-faire, savoir être), dans un souci de la professionnalisation des coach, autorisant cependant une grande latitude aux coachs dans l'exercice de leur « métier».

L'appartenance à une association professionnelle est considérée comme primordiale pour la reconnaissance du «métier »: «Déjà c'est un métier de personne seule, confronté à l'humain. C'est un métier où on est seul. Enrichir sa pratique, trouver des forums, des lieux d'échanges avec d'autres, relèvent de la nécessité » (entretien coach externe, 2011). L'objectif de se former, d'avoir des échanges de pratiques, des formations est crucial au regard d'un déficit de reconnaissance professionnelle :

Parce que c'est vrai que c'est une profession dans laquelle on a un talent rare pour exprimer très mal ce qu'on y fait. Ça sera intéressant d'ailleurs de creuser pourquoi. Qu'est ce qu'on fait pour aussi mal parler de nous. Bon, c'est intéressant. Et c'est une question qu'on se pose chez ICF (entretien coach externe, 2011).

La mauvaise perception de la profession de coach serait dûe à une communication déficiente, d'où l'importance d'adhérer à une association de coachs pour bénéficier de la vitrine des référentiels de compétences, des codes de déontologie, des certifications. Elle laisse entrevoir un certain flou de cette professionnalisation des coachs, qui renforcerait la nécessité du recours à l'éthique et à la déontologie.

Plusieurs moments dans la pratique des coachs et dans le déroulement du coaching peuvent, en effet, être délicats et sollicitent l'éthique des coachs ou leur déontologie. La question de la négociation de la demande et la question de la restitution au commanditaire sont évoquées dans la majorité des entretiens. Prenons l'exemple du respect de la confidentialité vis-à-vis du coaché. Celle-ci fait référence à des articles des codes de déontologie : dans le titre 2 du code de la SFCoach

\footnotetext{
«Devoirs du coach vis-à-vis du coaché - article 2.3: «Toute demande de coaching, lorsqu'il y a prise en charge par une organisation, répond à deux niveaux de demande : l'une formulée par l'entreprise et l'autre par l'intéressé lui-même. Le coach valide la demande du coaché ».
} 
Dans le titre 3 du code de la SFCoach, l'article 3.2 - Restitution au donneur d'ordre - stipule que « le coach ne peut rendre compte de son action au donneur d'ordre que dans les limites établies avec le coaché » (www.sfcoach.org, consulté le 26 avril 2016). Dans le code ICF, c'est l'article 4, décomposé en 4 sous-articles, qui prescrit une stricte confidentialité, les limites de celle-ci étant la loi (www.coachfederation.fr, consulté le 26 avril 2016).

Dans les entretiens, les coachs n'évoquent que très peu les codes de déontologie dans leur totalité et leur cohérence mais ils reprennent les «principes intangibles » que doit respecter le coach, principe de confidentialité, principe de supervision du coach etc. Certains font référence à la déontologie, comme cadre, comme délimitation des activités de coaching. C'est le cas de ce coach interne dans une collectivité territoriale, qui évoque ainsi ce que représente pour lui le code de déontologie : « Alors, le fameux cadre juridique, mais ce n'est pas du juridisme, c'est véritablement un bordage. Le coaching n'est pas une profession à ordre, y'a pas de code de déontologie donc le contrat5 pose des limites » (entretien consultant coach interne, 2011).

Ainsi, les codes de déontologie font plutôt partie intégrante des prestations offertes par les associations de coaching qui oeuvrent à la professionnalisation des coachs : le marketing de la profession passe par les référentiels de compétences, les accréditations des formations au coaching, les certifications ${ }^{6}$. Au titre des autres prestations, ils contribuent à distinguer le coach des « gourous » ou des « marchands de soupe ».

Alors, le phénomène gourou, moi c'est un problème d'éthique et de valeur personnelle, voilà, c'est alors... je pense que dans les fédérations, on travail beaucoup à ça, et chez ICF notamment... Mais je pense que si y'a ce risque là, il est minime, si les coachs en question ont un minimum d'éthique. Le coach il a la discrétion de se retirer gentiment... Le coach, dont on ne parle que de lui, là on est dans les phénomènes de gourou, ça existe bien entendu, y'en a, y'a même des gourous chez les coachs (entretien consultant coach interne, 2011).

\footnotetext{
${ }^{5}$ Il s'agit du contrat tripartite négocié au départ entre le coach, le commanditaire et le manager coaché ${ }^{6}$ Par exemple, pour être Master Coach chez ICF, il faut justifier de 2500 heures de pratiques et analyser dans le cadre de dossiers très lourds les expériences menées, dans les contextes du client, et les dossiers remis à la fédération par les consultants-coachs sont en général contrôlés auprès des clients, avant l'attribution de la certification.
} 
C'est donc l'éthique qui pallie la relative faible contrainte liée aux codes de déontologie. Dans les paroles sur les pratiques, c'est la référence à l'éthique qui est récurrente et prédominante. Quelle est cette éthique du coaching ?

\section{L'éthique de soi dans le coaching}

L'éthique de soi $^{7}$ dans le coaching, définirait une visée du «bien-vivre » en entreprise, fondée sur la valorisation (le développement) de la personne conçue comme un tout, selon une conception holistique revendiquée par les coachs. Les mots du néo-management (Boltanski, Chiapello, 1999) afférant à la personne (qui est le sujet du coaching) sont le respect, l'authenticité, la liberté, l'autonomie, la maitrise de soi, l'estime de soi, le dépassement de soi. Ces mots recoupent les valeurs morales au fondement de la responsabilisation du coaché, qui peuvent pour nous s'interpréter à travers la pensée de Ricoeur, lorsqu'il énonce : «L'expérience morale ne demande rien de plus qu'un sujet capable d'imputation, si l'on entend par imputabilité la capacité d'un sujet à se désigner comme l'auteur véritable de ses propres actes »; il s'agit bien «du vis-à-vis subjectif de la norme », que l'auteur nomme "liberté pratique » (Ricoeur, 1990). Le philosophe s'oppose ainsi à Kant, et «à l'expérience morale commune selon laquelle ne peuvent être tenues pour obligatoires que les maximes d'action, qui satisfont à un test d'universalisation » (Ricoeur, 1990). Ainsi, l'estime de soi, au cœur de la relation de coaching peut être considérée comme «l'interprétation de soi-même médiatisée par l'évaluation éthique de nos actions » (Ricoeur, 1990).

Cette éthique de soi dans le coaching légitime alors l'importance du rôle conféré à la psyché dans le dispositif et les nouvelles tendances managériales, comme celle de «l'entreprise de soi » (Aubrey, 2000). Les références8 de cette éthique de soi peuvent être trouvées dans le sport (le coach sportif), dans la philosophie (le surhomme de Nietzche), dans la religion (Krishna, le cocher du char d'Arjuna dans l'épopée hindouiste du Mahabarata, l'abbé Lanfranc, conseiller du prince Guillaume Le Conquérant), dans la littérature (Le prince de Machiavel), dans la psychologie (Le moi idéal renvoyant au narcissisme infantile et l'idéal du moi comme objectif à atteindre, chez Freud, repris par le psychosociologue Enriquez, 1997). Ces références orchestrent les compétences des coachs qui doivent avant tout savoir «écouter » et « donner du feed-back », pour transformer l'individu, responsable non seulement de ses performances mais de celles de ses équipes, quelque soit le contexte économique ou organisationnel. L'idéalisation façonne la représentation du personnage du coach,

\footnotetext{
${ }^{7}$ Le soi et l'autre comme deux notions corrélatives au sens de Ricoeur (1992)

${ }^{8}$ Références récurrentes dans la littérature managériale sur le coaching (Guilhaume, 2009)
} 
créant chez le coaché une forte adhésion, exprimée dans les entretiens. Et elle forge les objectifs du coaché, dans une quête du manager « hors limites » (Kerjean, 2000).

En conséquence, le coach, pour répondre à cet idéal du moi, place l'éthique au sommet des exigences professionnelles auxquelles il doit s'efforcer de répondre. C'est aussi plus prosaïquement son « humanisme », qui le conduit à introduire une « relation de confiance» dans le coaching, permettant au coaché de se confier. Ecoutons cette coach interne d'un grand groupe :

C'est la personne qui choisit ce qu'elle a envie de dire. C'est la base. C'est sacré, ce climat de confiance. Donc un espace de respiration un espace de liberté finalement ... Comme c'est bien borné, à l'intérieur du cadre on peut tout faire. Où la personne va pouvoir trouver elle-même ses solutions aux problèmes ou aux situations qu'elle amène. Donc ce sont des espaces de prise de recul, de questionnement, d'élaboration en fait de la personne. Voilà : moi ce que j'apporte c'est une position très haute sur le cadre, et très très basse sur le contenu (coache interne, 2012).

L'éthique, au carrefour du sacré et de l'humain, consiste pour le coach à se rendre « invisible pour ne pas créer une situation de dépendance avec le coaché » (coach externe, 2011).

Et c'est aussi l'intérêt qu'il attache à son propre développement personnel, qui permet au coach d'exercer de façon éthique :

Sur le qui êtes-vous plus globalement, je pourrais dire que j'essaye d'être exemplaire en termes d'acheminement personnel, de libération de fausses croyances, de réflexion sur moi- même, de trouver la personne que je suis vraiment, d'être unique, et que du coup, finalement cette réflexion hors de toute chapelle essaie d'être profitable pour mes clients, qu'il soit en coaching ou en thérapie (coach externe, 2011).

L'estime de soi ${ }^{9}$ permet alors de transmettre cette éthique par une communication qui fasse sens : les coachs indiquent travailler beaucoup sur les problématiques de communication :

Les gens demandent de plus en plus de sens, ils veulent comprendre. Avant on donnait un ordre et puis la personne obéissait, et faisait. Maintenant, la personne veut comprendre. Ce qui paraît normal. Donc il faut une communication qui soit plus adaptée (entretien coach externe, 2012).

L'analyse des entretiens montre que dans les pratiques des coachs, la question éthique est déclinée très différemment selon chacun d'entre eux : certains coachs interviewés dans l'enquête de thèse faisaient déjà état de la singularité de leurs

\footnotetext{
${ }^{9}$ L'interprétation de soi-même médiatisée par l'évaluation éthique de nos actions (Ricoeur, 1990).
} 
références (référence philosophique à l'aïkido, par exemple). Les consultants évoquent une éthique personnelle qui doit être reliée à la singularité des concepts et méthodes utilisées selon les coachs :

Donc, chacun a sa boîte à outils, si vous voulez, chacun a son histoire, a sa culture, moi par exemple, j'ai une formation plus de type sociologique que de type psychologique. Donc, je suis plutôt axé, moi, sur l'organisation. J'ai appris la psychologie après en formation professionnelle etc. Donc, c'est sûr que mes coachings vont être assez axés sur le contexte de l'organisation, la sociologie de l'entreprise et peut-être moins sur les ressorts individuels et de l'individu. D'autres confrères seront par exemple des psychologues du travail ou des psychologues cliniciens, ils seront plus axés sur la psychologie individuelle (coach externe, 2011).

L'éthique personnelle des coachs semble prédominer par rapport aux codes de déontologie qui sont interprétés et appliqués en fonction des profils et des références très variables des consultants.

\section{Ethique de soi et pouvoir d'agir : des paradoxes}

Dans les discours de professionnalisation des coachs, cette éthique de soi doit se transformer en "pouvoir d'agir » (empowerment) du coaché, seul responsable de la transformation de ses actions.

Mais l'éthique de soi (liberté du coaché dans la relation au coach par exemple) est parfois confrontée à la déontologie, qui considère tant les intérêts de l'entreprise que celui des coachés (art 3.3 du titre 3 « Les devoirs du coach vis-à-vis de l'organisation, SFCoach, version 2011). Les entretiens menés montrent que l'éthique de soi dans le coaching ne peut alors être que paradoxale : en effet, comment le coach humaniste peut-il contribuer au développement personnel de l'individu, voire à son épanouissement, lorsque l'entreprise exerce une forte pression sur lui, comme nous l'indique un consultant ? (consultant RH et coach, 2011) :

Je crois qu'il y a pas mal de pression sur le manager. Et que souvent, même si ce n'est pas dit, on lui fait comprendre que le coaching, c'est un peu une dernière chance avant de prendre des dispositions plus coercitives, voilà. C'est ce que je vous disais, cette évolution du coaching, qui, au début c'était vraiment accompagner quelqu'un pour développer son potentiel, maintenant, ça peut être aussi une démarche pour rectifier les comportements qui ne sont pas en phase avec ce qu'attend l'entreprise.

Le coach lui-même doit faire face à des difficultés :

La difficulté, c'est qu'on travaille vous l'avez compris, on travaille sur des modifications de comportement, donc de personnalité c'est très difficile hein de 
changer de personnalité. Alors j'allais dire à un certain âge, mais tout le temps d'ailleurs. Donc s'il n'y a pas une prise de conscience, et une très forte, au début, de la part du cadre qu'il va falloir nécessairement un changement ou une évolution, ben ça marche pas bien... Alors, la difficulté ça peut être aussi, depuis cinq ans ... on reçoit beaucoup de gens, quand ils ont des problèmes 'Allez, va faire du coaching ça va allez mieux' c'est ce qu'on appelle un peu un coaching contraint, c'est-à-dire la personne n'est pas forcement volontaire (coach externe, 2012).

C'est le comportement éthique du coach qui intervient alors dans le fameux entretien tripartite de démarrage,

qui peut durer une heure et demie, deux heures, où l'on va essayer de lever tous les lièvres, tel que quelqu'un à qui on a prescrit un coaching, mais qui n'en a pas vraiment envie, les coachings de dernière chance, 'vous lui faites un coaching : il réussit, sinon il est viré, enfin il est pas viré chez nous, mais il est enlevé de son poste', donc, ça, on essaye de le lever, au moins que ça soit mis sur la table, parce qu'il y a des fois où ça arrive mais il faut le savoir ... il faut vérifier que c'est le volontariat et poser les protections par rapport à tout ce qui, effectivement pourrait arriver en terme d'abus d'influence (coach externe, 2012).

Enfin, le coaching interne suppose que le coach n'ait pas de positionnement hiérarchique par rapport au coaché, alors qu'il est dans la même structure :
Le coaching ne peut pas exister comme moyen de contrôle. Il ne peut pas y avoir coaching qui soit à la fois du développement pour la personne et pour l'organisation. Le coaching existe en plus des compétences du coach, que si il y a une réflexion éthique, menée par l'entreprise qui laisse effectivement une place au coaching (coach interne, 2012).

Dans ce verbatim, on observe que l'éthique prime sur la déontologie (article $2.3 \mathrm{du}$ code SFCoach, ci-dessus), et que l'éthique du coach peut être confrontée à celle de l'organisation.

L'analyse de J.E.Grunig (2014) concernant l'éthique dans les relations publiques est très éclairante sur ce point et peut s'appliquer aux professionnels du coaching, qui, comme nous l'avons montré, sont avant tout des communicants. En effet, « Le thème de la loyauté partagée est donc au cœur des questions éthiques relatives à la pratique des relations publiques. Deux concepts, soit les rôles sociaux et les valeurs des relations publiques, peuvent aider à résoudre ces problèmes. Face à des conflits de loyauté, les praticiens des relations publiques reviennent souvent à leurs croyances élémentaires au sujet du monde qui les entoure (leur vision du monde) afin de partager le bien du mal. Selon cet auteur, les coachs rencontrés seraient plutôt dans une perspective idéaliste :

Ceux qui se placent dans la perspective idéaliste adoptent une approche symétrique dans leur pratique des relations publiques. Selon les praticiens de ce groupe, les 


\begin{abstract}
relations publiques doivent servir à la fois les intérêts du public et ceux des organisations, contribuer à la tenue de débats informés sur les enjeux sociaux et faciliter le dialogue entre les organisations et leurs publics. Alors que les praticiens de la catégorie radicale utilisent les relations publiques de manière à orienter les changements sociaux dans la direction qu'ils privilégient, les praticiens de la catégorie idéaliste croient que la société se forme grâce au dialogue et à la résolution des conflits entre groupes opposés (Grünig, 2007).
\end{abstract}

Cette perspective idéaliste inscrite dans les codes de déontologie, placent les coachs dans des injonctions paradoxales qui peuvent rétroagir sur l'éthique elle-même, qui devient paradoxale. Dans le verbatim qui suit, la consultante coach externe exprime bien sa pratique de communication paradoxale, correspondant à cette perspective idéaliste :

Le coaché a le choix, qu'on peut tout de suite arrêter, tout de suite, car cette liberté a un coût et on peut regarder ça avec eux, parce que dans le cas d'un coaching prescrit, il y a un coût par rapport à la structure, 'Je vous offre un coaching, vous le refusez ? Très bien, la structure en prend acte, en prend note', donc c'est pas neutre. Tout acte dans l'organisation n'est pas neutre donc, moi je leur dis tout de suite, je mets les pieds dans le plat, voilà on vous a obligé, la bonne nouvelle, c'est qu'on peut arrêter tout de suite et on peut regarder les conséquences que ça aura. Maintenant regardons ce truc dont ce cadre qu'on vous a imposé, et qui peut devenir tout à coup un cadre où il y aurait de la liberté malgré tout (caoch externe, 2011).

Et c'est l'éthique qui est appelée à rescousse dans ce cas :

Dans la pratique, il se peut, dans certains cas que la hiérarchie impose un coaching. Après ça va dépendre de l'éthique du coach, de sa propre éthique personnelle, au niveau d'ICF en tout cas et à mon niveau personnel en tout cas, pour moi c'est rédhibitoire (coach externe, 2011).

Les coachs doivent résoudre d'autres paradoxes, comme celui d'une relation de coaching basée sur la parole intime, mais, se distinguant de la psychothérapie. Dans les entretiens, les coachs définissent un travail en coaching qui s'effectue sur le comportement, sur la personnalité, sur la construction de l'individu maintenant et dans l'immédiat et non sur l'analyse du passé par un travail sur l'inconscient. De même, la clause du secret professionnel (article 1.2 «Confidentialité » du titre I «Devoirs du coach », code SFCoach, version 2011) suppose que les éléments de restitution au commanditaire soient négociés avec le coaché ; il n’y a pas de rapport écrit, les coachs indiquant cependant restituer au commanditaire les tests de personnalité effectués durant la première phase du coaching. Ceci correspond à l'évaluation du coaching, qui reste floue en général, une obligation de moyens, mais pas de résultats : c'est l'article 1.5 «Obligations de moyens » du titre « Devoirs du coach » (Code SFCoach, version 2011): l'évaluation, elle, porte essentiellement sur les changements de 
comportements observés par la hiérarchie, par les collègues : l'entretien $360^{\circ 10}$ peut être utilisé dans certaines entreprises pour évaluer les effets du coaching.

Comment ces paradoxes éthiques agissent sur le pouvoir de décision des coachs et des managers coachés ? Notre première enquête, fondée à part égale sur des entretiens auprès des coachs et des coachés indique que les actions mises en œuvre (actions de communication participative des équipes, action de «changer son style de communication ») par les managers ont connu assez rapidement un essoufflement, dû à la pression productive, au contexte de changement et de restructuration permanents, conduisant les managers à se soumettre à des injonctions paradoxales. Cependant, le coaching transmettrait bien aux managers un pouvoir de communication, qui consisterait à euphémiser le pouvoir de décision économique du sommet, rendant parfois les coachs et les salariés « responsables » de choix imposés : c'est le cas du manager qui organise une communication participative auprès de ses équipes, pour faire accepter des mobilités forcées (Guilhaume, 2009). Les enquêtes plus récentes confirment cette analyse et notamment la difficulté à faire changer les comportements :

Bah, c'est-à-dire que le cadre lui-même, par exemple, un cadre, on va lui dire : 'tu mets trop de pression dans ton management', mais lui- même en subit trop de la part de son environnement. En ce moment, voilà, je fais un coaching à un directeur financier dans une grosse entreprise, on lui met clairement des bâtons dans les roues. C'est pour ça que d'ailleurs, que dans ce cas, on va remonter à un niveau de coaching collectif pour confronter les cadres qui sont en train de se retirer un peu... (consultant RH et coach externe, 2011)

Face à ces paradoxes, le professionnalisme des coachs résiderait ainsi dans leur capacité éthique à exercer une «sagesse pratique » au sens de Ricoeur ; comme le montre très bien A. Marcel Madila Basanguka, il y a chez le philosophe Ricoeur un « tragique à l'origine de l'éthique », c'est-à-dire que « la poésie explore au plan de la fiction le jeu des relations fondamentales entre la vie, la mort, le bien et le mal » (Ricoeur11, 1990, cité par Basanguka, 2005) ; mais, le conflit n'existe pas qu'à l'origine de l'éthique mais au terme de la réflexion morale,

car ce sont les conflits mêmes suscités par le passage nécessaire de la visée de la vie bonne par les fourches caudines de la norme, selon les trois étapes de l'estime de soi, de la sollicitude et du sens de la justice, qui conduisent au recours à la vertu aristotélicienne de la phronèsis ou sagesse pratique. Par sagesse pratique on entend, depuis le Stagirite, la capacité de jugement qui assure l'application de la norme

\footnotetext{
${ }^{10}$ Entretien d'évaluation du salarié circulaire, fondé essentiellement sur des critères de savoir être, et où interviennent hiérarchie, collègues, subordonnés, clients etc.

${ }^{11}$ Postface in Lenoir F., Le temps de la responsabilité, 1990 (voir bibliograhie)
} 
morale en accord avec une situation singulière et pour laquelle la conviction, déjà 'instruite' par le sens éthique le plus originaire, est plus décisive que la règle ellemême (Ricoeur, 1991, cité par Basanguka, 2005).

Le professionnalisme des coachs ne peut être séparé de leurs convictions au-delà des codes de déontologie, qui ne forgent une «sagesse pratique » qu'à condition de se préserver d'une servitude volontaire, s'accommodant plus ou moins bien des paradoxes du management de dirigeants reportant leurs responsabilités sur les coachs et les managers, censés faire passer les décisions difficiles par une communication euphémisée.

Cette analyse des jeux entre éthique de soi et déontologie dans les discours et les pratiques du coaching s'inscrit plus largement dans le rôle de l'éthique dans la communication et le management des organisations et entreprises. Celle-ci jouerait un rôle fondamental de légitimation des professionnels de la communication et du coaching, dans un «esprit du capitalisme » (Boltanski, Chiapello, 1999), où seul l'éthique, issue de l'expérience personnelle, permet de faire face aux paradoxes largement décrits. Comme le montre R. Huet, l'éthique et la RSE seraient une des réponses des entreprises face aux dysfonctionnements sociaux actuels (Huet, 2012, p. $25)$; il souligne le fait que l'éthique s'objective dans des écrits qui sont l'œuvre d'individus (ibid, p. 38). L'éthique, au cœur de la responsabilité individuelle des coachs et communicants contribuerait ainsi à une nouvelle régulation sociale d'entreprise, au regard de l'affaiblissement des médiations traditionnelles. 


\section{Bibliographie}

Aubrey B. (2000). L'entreprise de soi. Paris : Flammarion.

Basanguka Marcel Madila A. (2005). Ethique et imagination chez Paul Ricoeur. Revue d'éthique et de théologie morale, $\mathrm{n}^{\circ} 233$, p. 113-134.

Boltanski L., Chiapello E. (1999). Le nouvel esprit du capitalisme. Paris : Gallimard.

Brunel V. (2008). Les managers de l'âme, le développement personnel en entreprise, une nouvelle pratique de pouvoir ? Paris : La découverte.

Enriquez E. (1997). Les jeux du pouvoir et du désir dans l'entreprise. Paris : Desclée de Brouwer.

Grünig James A. (2014). Questions et théorie en éthique des relations publiques. Communiquer, $\mathrm{n}^{\circ} 11$

Guilhaume G. (2009). L'ère du coaching, critique d'une violence euphémisée. Paris : Syllepse.

Guilhaume G. (2013). Le coaching et l'entretien annuel, une visibilité en 'blanc et noir' de l'individu. Communication et organisation, $n^{\circ} 44$, p. 99-110. Pessac : Grec/o - Université Michel de Montaigne, Bordeaux 3.

Huet R. (2012). La fabrique de l'éthique. Les nouvelles promesses des entreprises. Paris : CNRS Editions.

Kerjean A. (2000). Les nouveaux comportements en entreprise, oser secouer l'organigramme. Paris : Editions d'Organisation.

Lenhardt V. (2015). Les responsables porteurs de sens, Culture et pratique du coaching et du team building. Paris : Eyrolles.

Maas E. (2011). Culture managériale, versus culture organisationnelle. Une tension contre-productive. Dans Kaciaf N., Legrave J.B. (dir.), Communication interne et changement. Paris : L'Harmattan.

Persson S., Rappin B. (2013). Il était une fois le coaching. Humanisme et entreprise, $\mathrm{n}^{\circ} 311$, p. 41-60.

Pinson G., Sala Pala V. (2007). Peut-on vraiment se passer de l'entretien en sociologie de l'action publique ? Revue française de sciences politiques, vol 57, p. 555-597. Paris : Presses de Sciences Po.

Ricœur P. (1990). Postface. Dans Lenoir F. Le Temps de la responsabilité, p. 249270. Paris : Fayard.

Ricoeur P. (1990). Ethique et morale. Revue de l’Institut catholique de Paris, $n^{\circ} 34$. 
26 Professionnalisation de la communication : approches sectorielles

Ricoeur P. (1990). De la morale à l'éthique et aux éthiques. www.mapageweb.umontreal.ca/lepagef/dept/.../Ricoeur_MORALE.pdf

Ricoeur P. (1990). Soi-même comme un autre, Paris : Seuil.

Ricoeur P. (1991). Lectures 1, Autour du politique. Paris : Seuil.

Ricoeur P. (1992). Lectures 2, La contrée des philosophes, Paris : Seuil.

Zarifian P. (2010). La communication dans le travail. Communication et Organisation, $\mathrm{n}^{\circ}$ 38, p. 135-146. Pessac: Grec/o - Université Michel de Montaigne, Bordeaux 3. 


\section{Annexe 1}

Le code de déontologie de la SFcoach (texte intégral. V 2011)

\section{Titre 1 - Devoirs du coach}

\section{Art. 1-1 - Exercice du Coaching}

Le coach s'autorise en conscience à exercer cette fonction à partir de sa formation, de son expérience et de sa supervision.

Art. 1-2 - Confidentialité

Le coach s'astreint au secret professionnel.

Art. 1-3 - Supervision établie

L'exercice professionnel du coaching nécessite une supervision. Les Membres accrédités de la Société Française de Coaching sont tenus de disposer d'un lieu de supervision.

Art. 1-4 - Respect des personnes

Conscient de sa position, le coach s'interdit d'exercer tout abus d'influence.

Art. 1-5 - Obligation de moyens

Le coach prend tous les moyens propres à permettre, dans le cadre de la demande du client, le développement professionnel et personnel du coaché, y compris en ayant recours, si besoin est, à un confrère.

Art. 1-6 - Refus de prise en charge

Le coach peut refuser une prise en charge de coaching pour des raisons propres à l'organisation, au demandeur ou à lui-même. Il indique dans ce cas un de ses confrères.

\section{Titre 2 - Devoirs du coach vis à vis du coaché}

Art. 2-1 - Lieu du Coaching 
Le coach se doit d'être attentif à la signification et aux effets du lieu de la séance de coaching.

Art. 2-2 - Responsabilité des décisions

Le coaching est une technique de développement professionnel et personnel. Le coach laisse de ce fait toute la responsabilité de ses décisions au coaché.

Art. 2-3 - Demande formulée

Toute demande de coaching, lorsqu'il y a prise en charge par une organisation, répond à deux niveaux de demande : l'une formulée par l'entreprise et l'autre par l'intéressé lui-même. Le coach valide la

demande du coaché.

Art. 2-4 - Protection de la personne

Le coach adapte son intervention dans le respect des étapes de développement du coaché.

\section{Titre 3 - Devoirs du coach vis à vis de l'organisation}

Art. 3-1 - Protection des organisations

Le coach est attentif au métier, aux usages, à la culture, au contexte et aux contraintes de l'organisation pour laquelle il travaille.

Art. 3-2 - Restitution au donneur d'ordre

Le coach ne peut rendre compte de son action au donneur d'ordre que dans les limites établies avec le coaché.

Art. 3-3 - Equilibre de l'ensemble du système

Le coaching s'exerce dans la synthèse des intérêts du coaché et de son organisation. 


\section{Titre 4 - Devoirs du coach vis à vis de ses confrères}

Art. 4-1-1 - Les Membres Postulants peuvent, dans toute communication professionnelle les concernant, faire état de leur "engagement écrit à respecter la Déontologie de la Société Française de Coaching".

Art. 4-1-2 - Selon l'accréditation qu'ils ont reçue, les autres membres ont le droit d'utiliser les appellations déposées ci-dessous dans toute communication professionnelle les concernant :

- pour les membres Titulaires : «membre Titulaire de la SFCoach®» (logo déposé)

- pour les membres Associés : «membre Associé de la SFCoach® » (logo déposé)

Art. 4-1-3 - Les droits ci-dessus sont conditionnés au versement effectif par le Membre concerné de sa cotisation annuelle appelée.

Art. 4-2 Obligation de réserve

Le coach se tient dans une attitude de réserve vis à vis de ses confrères.

\section{Titre 5 - Recours}

\section{Art. 5-1 - Recours auprès de la SF Coach®}

Toute organisation ou personne peut recourir volontairement auprès de la Société Française de Coaching en cas de manquement aux règles professionnelles élémentaires inscrites dans ce code ou de conflit avec un coach de la SF Coach®.

Une voie de dialogue et de recours

À l'instar d'un conseil de l'ordre, la SFCoach offre une instance de dialogue, de protection et de recours pour les entreprises, les médias, les institutions, les coachs et les coachés :

- Elle accueille les questions de chacun et contribue à la clarification de l'offre de coaching professionnel,

- Elle développe des partenariats avec diverses institutions, pour enrichir sa réflexion et promouvoir de "bonnes pratiques",

- Elle peut être saisie en cas d'éventuels conflits et intervenir à titre de médiateur et/ou de manière disciplinaire vis-à-vis de ses membres (jusqu'à leur exclusion) 


\section{Annexe 2}

Le référentiel de compétences de coachs : www.sfcoach.org : consultation le 10 mai 2016

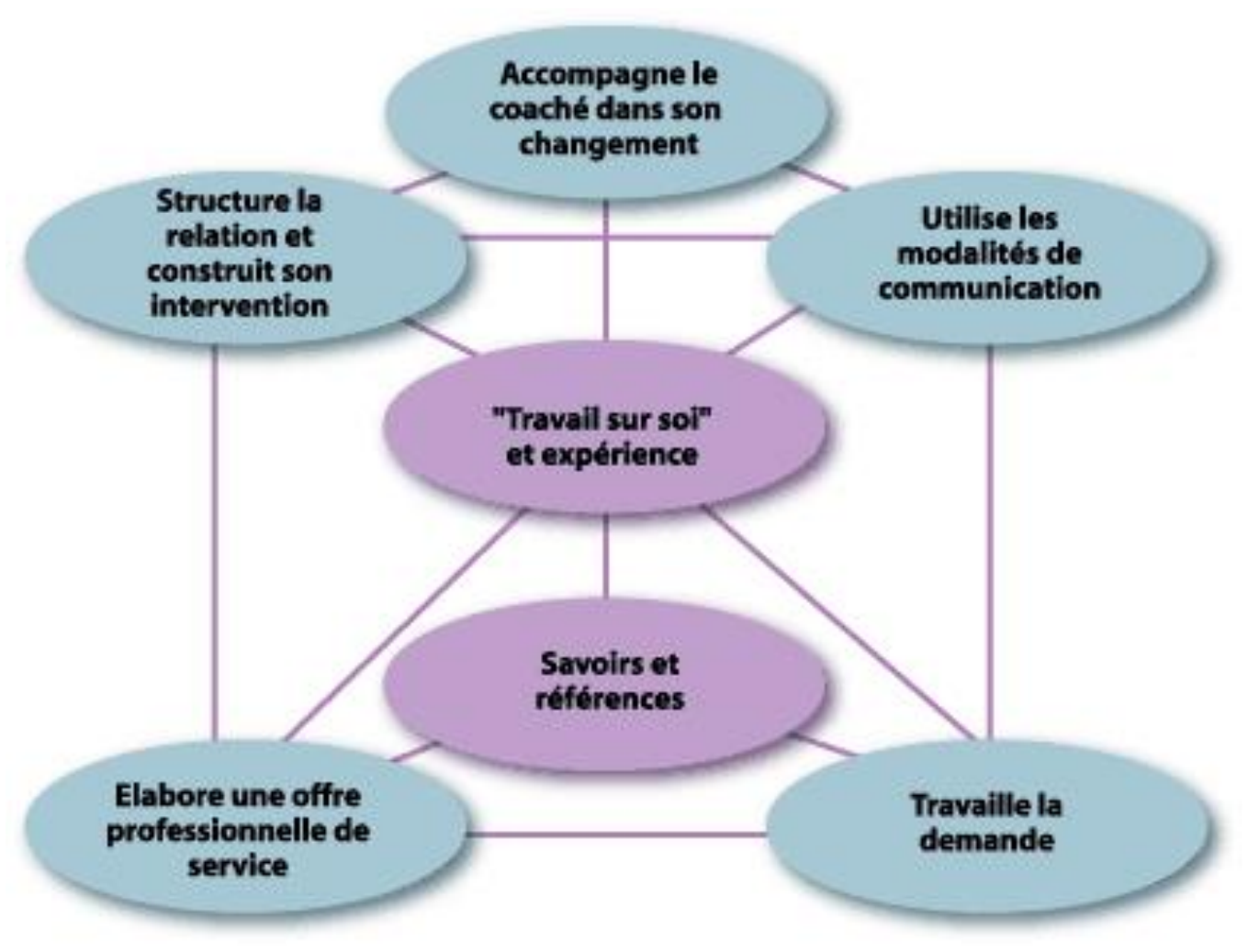

